\title{
LINE FORCES AND DISLOCATIONS IN ANGULARLY INHOMOGENEOUS ANISOTROPIC ELASTIC WEDGES AND SPACES*
}

\author{
BY
}

T. C. T. TING ${ }^{1}$

University of Illinois at Chicago

I. Introduction. In a fixed rectangular coordinate system $x_{i}(i=1,2,3)$, let the stress-strain laws of the anisotropic elastic material be written as

$$
\sigma_{i j}=C_{i j k s}(\theta) u_{k, s},
$$

in which $\sigma_{i j}$ is the stress, $u_{k}$ the displacement, a comma stands for differentiation, repeated indices imply summation, and $C_{i j k s}$ are the elastic stiffnesses which depend on the angle $\theta$ in the cylindrical coordinates:

$$
x_{1}=r \cos \theta, \quad x_{2}=r \sin \theta, \quad x_{3}=z .
$$

As in the homogeneous materials, $C_{i j k s}$ are assumed to have the symmetry property

$$
C_{i j k s}=C_{j i k s}=C_{i j s k}=C_{k s i j} \text {. }
$$

We will consider two-dimensional deformations in which $u_{i}(i=1,2,3)$ and hence $\sigma_{i j}$ are independent of $x_{3}$. Two problems are studied here. In the first problem, the infinite space is subject to a concentrated force and a straight line dislocation at the origin $r=0$. In the second problem, a wedge of wedge angle $\alpha$ occupying the region $0 \leq \theta \leq \alpha$ is subject to a concentrated force at the wedge apex $r=0$ while the sides of the wedge are traction free. Both problems have been studied for homogeneous anisotropic elastic materials (see [1-8], for example). In the case of homogeneous materials, Stroh's complex formalism is invariably employed and the final solutions can be expressed in real form for the displacement gradient $u_{k, s}$. The stress, however, is determined indirectly from (1). For the inhomogeneous material considered here, Stroh's formalism does not apply. We will see in this paper that one can derive the solutions in real form without introducing complex variables. The derivations of course remain valid for homogeneous materials. The solutions for displacement as well as for stress are all explicit and are surprisingly simple. We will also see that differentiations of $C_{i j k s}(\theta)$ with respect to $\theta$ are not called for in the derivations and therefore $C_{i j k s}(\theta)$ need not be continuous in $\theta$. This means that the results obtained here apply to composite spaces which may consist of two half-spaces

*Received January 22, 1988.

'Department of Civil Enginerring, Mechanics, and Metallurgy, University of Illinois at Chicago, Box 4348, Chicago, IL 60680 
of different homogeneous anisotropic materials $[9,10]$ or, more generally, a finite number of homogeneous anisotropic elastic wedges of different wedge angles [11]. In fact, the approach employed here was inspired by the form of solutions obtained for composite spaces in [11]. Unlike the derivations in [11] which employ Stroh's sophisticated complex formalism and several sum rules involving Stroh's eigenvalues and eigenvectors, the derivations presented here are elementary and require no sum rules.

Finally, it should be mentioned that the associated problem of angularly inhomogeneous isotropic elastic wedges has been investigated recently in [12].

2. Basic solutions. The equations of equilibrium are

$$
\sigma_{i j, j}=0 .
$$

For the problem under consideration, dimensional analyses show that the stress must be proportional to $r^{-1}$ in order to balance the applied concentrated force. Let

$$
\sigma_{i j}=r^{-1} \tau_{i j}(\theta),
$$

where $\tau_{i j}$ depends on $\theta$ only. Substituting into (4) and noticing that

$$
r, j=n_{j}, \quad \theta,{ }_{j}=r^{-1} m_{j}, \quad m_{j, \theta}=-n_{j},
$$

where the vectors $\mathbf{n}$ and $\mathbf{m}$ are

$$
\mathbf{n}=(\cos \theta, \sin \theta, 0), \quad \mathbf{m}=(-\sin \theta, \cos \theta, 0),
$$

we obtain

$$
\left(\tau_{i j} m_{j}\right)_{, \theta}=0
$$

Hence

$$
\tau_{i j} m_{j}=\frac{1}{\pi} g_{i}
$$

where $\mathbf{g}$ is a constant vector.

The surface traction vector $t_{\theta}$ on a radial plane $\theta=$ constant is

$$
\left(\mathbf{t}_{\theta}\right)_{i}=\sigma_{i j} m_{j}=r^{-1} \tau_{i j} m_{j}=\frac{1}{\pi r} g_{i}
$$

i.e.,

$$
\mathbf{t}_{\theta}=\frac{1}{\pi r} \mathbf{g}
$$

Thus the surface traction $\mathbf{t}_{\theta}$ is independent of $\theta$, an interesting fact which was first observed in [11].

For the displacement $\mathbf{u}$, we assume that

$$
\mathbf{u}=-\frac{1}{\pi}(\ln r) \mathbf{h}+\boldsymbol{\psi}(\theta)
$$

where $\mathbf{h}$ is a constant and $\psi$ depends on $\theta$ only. With (10), (1) leads to

$$
\sigma_{i j}=r^{-1}\left\{-\frac{1}{\pi} C_{i j k s} n_{s} h_{k}+C_{i j k s} m_{s} \psi_{k, \theta}\right\}
$$


which provides the expression for $\tau_{i j}$ in (5). Substituting $\tau_{i j}$ in (8b) we obtain the differential equation for $\psi(\theta)$,

$$
-\frac{1}{\pi} C_{i j k s} m_{j} n_{s} h_{k}+C_{i j k s} m_{j} m_{s} \psi_{k, \theta}=\frac{1}{\pi} g_{i}
$$

or, in matrix notation,

$$
-\frac{1}{\pi} \mathbf{R}^{\mathrm{T}} \mathbf{h}+\mathbf{T} \boldsymbol{\psi}, \theta=\frac{1}{\pi} \mathbf{g} .
$$

The superscript $\mathrm{T}$ denotes the transpose and

$$
\left.\begin{array}{l}
T_{i k}(\theta)=C_{i j k s} m_{j} m_{s}, \\
R_{i k}(\theta)=C_{i j k s} n_{j} m_{s}, \\
Q_{i k}(\theta)=C_{i j k s} n_{j} n_{s} .
\end{array}\right\}
$$

The matrix $\mathbf{Q}$ did not appear in (12) but will be needed below. It should be pointed out that $\mathbf{T}$ and $\mathbf{Q}$ are symmetric and, subject to positiveness of strain energy, positive definite. We rewrite (12) as

$$
\boldsymbol{\psi}_{, \theta}=\frac{1}{\pi}\left\{\mathbf{N}_{2}(\theta) \mathbf{g}-\mathbf{N}_{1}(\theta) \mathbf{h}\right\},
$$

in which

$$
\left.\begin{array}{l}
\mathbf{N}_{1}(\theta)=-\mathbf{T}^{-1} \mathbf{R}^{\mathrm{T}}, \\
\mathbf{N}_{2}(\theta)=\mathbf{T}^{-1}=\mathbf{N}_{2}^{\mathrm{T}}(\theta), \\
\mathbf{N}_{3}(\theta)=\mathbf{R T}^{-1} \mathbf{R}^{\mathrm{T}}-\mathbf{Q}=\mathbf{N}_{3}^{\mathrm{T}}(\theta) .
\end{array}\right\}
$$

We see that $\mathbf{N}_{2}$ and $\mathbf{N}_{3}$ are symmetric and $\mathbf{N}_{2}$ is positive definite. Again $\mathbf{N}_{3}$ did not appear in (14) but will be needed later on. If we define

$$
\left.\begin{array}{l}
\mathbf{S}(\theta)=\frac{1}{\pi} \int_{0}^{\theta} \mathbf{N}_{1}(\omega) d \omega, \\
\mathbf{H}(\theta)=\frac{1}{\pi} \int_{0}^{\theta} \mathbf{N}_{2}(\omega) d \omega, \\
\mathbf{L}(\theta)=\frac{1}{\pi} \int_{0}^{\theta}-\mathbf{N}_{3}(\omega) d \omega,
\end{array}\right\}
$$

integration of (14) provides the function $\psi$ :

$$
\boldsymbol{\psi}(\boldsymbol{\theta})=\mathbf{H}(\theta) \mathbf{g}-\mathbf{S}(\theta) \mathbf{h}+\mathbf{u}_{0},
$$

where $\mathbf{u}_{0}$ is an arbitrary constant. Noticing that $\mathbf{u}_{0}$ represents a rigid body motion, we may ignore $u_{0}$ and the displacement given by (10) has the expression

$$
\mathbf{u}=-\frac{1}{\pi}(\ln r) \mathbf{h}-\mathbf{S}(\theta) \mathbf{h}+\mathbf{H}(\theta) \mathbf{g}
$$

which contains two arbitrary constants $\mathbf{g}$ and $\mathbf{h}$.

The stress is best expressed in its cylindrical components. Since $\mathbf{m}, \mathbf{n}$, and $(0,0,1)$ are, respectively, the unit normal vector and the two unit tangential vectors to the radial plane $\theta=$ constant, we have by $(9 \mathrm{~b})$,

$$
\sigma_{\theta \theta}=\frac{1}{\pi r} \mathbf{m} \cdot \mathbf{g}, \quad \sigma_{\theta r}=\frac{1}{\pi r} \mathbf{n} \cdot \mathbf{g}, \quad \sigma_{\theta 3}=\frac{1}{\pi r} g_{3} .
$$

On the other hand, the surface traction $\mathbf{t}_{r}$ on the surface $r=$ constant is

$$
\left(\mathbf{t}_{r}\right)_{i}=\sigma_{i j} n_{j} \text {, }
$$


which is, making use of (11), (13), (14), and (15),

$$
\mathbf{t}_{r}=\frac{1}{\pi r}\left\{\mathbf{N}_{3}(\theta) \mathbf{h}-\mathbf{N}_{1}^{\mathrm{T}}(\theta) \mathbf{g}\right\} .
$$

The stresses on the surface $r=$ constant are given by

$$
\sigma_{r r}=\mathbf{n} \cdot \mathbf{t}_{r}, \quad \sigma_{r \theta}=\mathbf{m} \cdot \mathbf{t}_{r}, \quad \sigma_{r 3}=\left(\mathbf{t}_{r}\right)_{3} .
$$

It can be shown that $\sigma_{r \theta}$ obtained from (20a) and $(20 b)_{2}$ is identical to $\sigma_{\theta r}$ obtained from $(19)_{2}$ if we use the identities [13]

$$
\mathbf{N}_{3}(\theta) \mathbf{m}(\theta)=\mathbf{0}, \quad \mathbf{N}_{1}(\theta) \mathbf{m}(\theta)=-\mathbf{n}(\theta) .
$$

Although identities (21) are proved in [13] for homogeneous materials, it is not difficult to see from (15) and (13) that the identities hold here also.

We have thus obtained basic solutions for displacement and stress given by (18), (19), and (20). The solutions are in real form and contain arbitrary constants $\mathbf{g}$ and $\mathbf{h}$ which will be determined by the boundary conditions.

3. Infinite space subject to a force and a dislocation. Consider the infinite space which is subject to a concentrated force $f$ and a line dislocation with the Burgers vector $\mathbf{b}$ at the origin. To balance the force $\mathbf{f}$, we must have

$$
-\int_{0}^{2 \pi} r \mathbf{t}_{r}(\theta) d \theta=\mathbf{f}
$$

or, using (20a) and $(16)_{1,3}$,

$$
\mathbf{S}^{\mathrm{T}}(2 \pi) \mathbf{g}+\mathbf{L}(2 \pi) \mathbf{h}=\mathbf{f} .
$$

The difference in the displacements at $\theta=0$ and $2 \pi$ must be equal to the Burgers vector $\mathbf{b}$ and we have from (18),

$$
\mathbf{H}(2 \pi) \mathbf{g}-\mathbf{S}(2 \pi) \mathbf{h}=\mathbf{b} .
$$

Elimination of $\mathbf{h}$ or $\mathbf{g}$ between (23) and (24) leads to

$$
\begin{aligned}
& \mathbf{g}=\mathbf{U}^{-1}\left\{\mathbf{S}(2 \pi) \mathbf{L}^{-1}(2 \pi) \mathbf{f}+\mathbf{b}\right\}, \\
& \mathbf{h}=\mathbf{V}^{-1}\left\{\mathbf{f}-\mathbf{S}^{\mathrm{T}}(2 \pi) \mathbf{H}^{-1}(2 \pi) \mathbf{b}\right\},
\end{aligned}
$$

where

$$
\left.\begin{array}{l}
\mathbf{U}=\mathbf{H}(2 \pi)+\mathbf{S}(2 \pi) \mathbf{L}^{-1}(2 \pi) \mathbf{S}^{\mathrm{T}}(2 \pi), \\
\mathbf{V}=\mathbf{L}(2 \pi)+\mathbf{S}^{\mathrm{T}}(2 \pi) \mathbf{H}^{-1}(2 \pi) \mathbf{S}(2 \pi) .
\end{array}\right\}
$$

It is clear that $\mathbf{H}(\theta)$ defined in $(16)_{2}$ is positive definite because the integrand $\mathbf{N}_{2}(\omega)$ is the inverse of $\mathbf{T}$ according to $(15)_{2}$ which is positive definite. Hence $\mathbf{H}^{-1}$ exists. As to $\mathrm{L}(\theta)$ of $(16)_{3}$, we consider

$$
\mathbf{y}^{\mathrm{T}} \mathbf{L}(\theta) \mathbf{y}=\frac{1}{\pi} \int_{0}^{\theta} \mathbf{y}^{\mathrm{T}}\left\{-\mathbf{N}_{3}(\omega)\right\} \mathbf{y} d \omega,
$$

where $\mathbf{y}$ is a $3 \times 1$ constant matrix. Following the proof in [14], it can be shown that $-\mathbf{N}_{3}(\omega)$ is positive semi-definite and that the integrand in $(28)$ is positive and nonzero except possibly at two values of $\omega$ for which the integrand vanishes. Hence $\mathbf{L}(\theta)$ is positive definite and its inverse exists. With $\mathbf{H}(\theta)$ and $\mathbf{L}(\theta)$ being positive definite, it is clear from (27) that $\mathbf{U}$ and $\mathbf{V}$ are positive definite. Consequently, $\mathbf{U}^{-1}$ and $\mathbf{V}^{-1}$ exist. 
4. Wedge subject to a concentrated force. We now consider the case of a wedge of wedge angle $\alpha$ which occupies the region $0 \leq \theta \leq \alpha$. The wedge is subject to a concentrated force $\mathbf{f}$ at the wedge apex $r=0$ while the sides $\theta=0, \alpha$ are traction free. From $(9 b)$ we see that

$$
\mathbf{g}=\mathbf{0},
$$

and use of (20a) with $\mathbf{g}=\mathbf{0}$ in the following balance equation

$$
-\int_{0}^{\alpha} r \mathbf{t}_{r}(\theta) d \theta=\mathbf{f}
$$

leads to

$$
\mathbf{L}(\alpha) \mathbf{h}=\mathbf{f},
$$

or,

$$
\mathbf{h}=\mathbf{L}^{-1}(\alpha) \mathbf{f}
$$

We see that surface traction $\mathbf{t}_{\theta}$ vanishes not only at $\theta=0, \alpha$, but on any radial plane (see also $[7,11])$.

5. Concluding remarks. With the constants $\mathbf{g}$ and $\mathbf{h}$ given by (25), (26) for the infinite space and by (29), (31) for the wedge, (18) provides the solution for displacement and (19), (20) the solution for stress. In the case of composite spaces or wedges, $\mathbf{T}, \mathbf{R}, \mathbf{Q}$ defined in (13) are piecewise continuous in $\theta$ and so are $\mathbf{N}_{i}$ $(i=1,2,3)$ of $(15)$. This presents no problems in the integrals in (16) and the results recover that obtained in [11].

The only stress component not presented here is $\sigma_{33}$ which can be determined from (1) as

$$
\sigma_{33}=C_{33 i j} u_{i, j}
$$

To find the displacement gradient $u_{i, j}$ we obtain from (18), (6), and (16),

$$
u_{i, j}=-\frac{1}{\pi r}\left\{h_{i} n_{j}+\left(\mathbf{N}_{1}\right)_{i k} h_{k} m_{j}-\left(\mathbf{N}_{2}\right)_{i k} g_{k} m_{j}\right\} .
$$

Letting $G_{i j}=C_{33 i j}$, we have

$$
\sigma_{33}=-\frac{1}{\pi r}\left\{\mathbf{n}^{\mathrm{T}} \mathbf{G h}+\mathbf{m}^{\mathrm{T}} \mathbf{G}\left[\mathbf{N}_{1} \mathbf{h}-\mathbf{N}_{2} \mathbf{g}\right]\right\} .
$$

Addendum. Since the acceptance of this paper we have seen a note by Kirchner [15] who studied the same problem presented here. As it should be the case, many results obtained here agree with that in [15]. In particular, Eq. (9b) which shows that the surface traction $\mathbf{t}_{\theta}$ on any radial plane $\theta=$ constant is independent of $\theta$ was also observed by Kirchner. It should be pointed out however that the approach used in [15] is quite different from that employed here. Specifically, Eq. (9b) derived here made no use of the constitutive laws of the materials. Therefore, as long as $\sigma_{i j}$ is proportional to $r^{-1}, \mathbf{t}_{\theta}$ is independent of $\theta$ regardless of whether the material is isotropic or anisotropic, homogeneous or heterogeneous, elastic or inelastic, linear or nonlinear. A similar phenomenon was observed for elastic wedges subject to a concentrated couple [14]. 


\section{REFERENCES}

[1] J. D. Eshelby, W. T. Read, and W. Shockley, Anisotropic elasticity with applications to dislocation theory, Acta Metall. 1, 251-259 (1953)

[2] A. N. Stroh, Dislocations and cracks in anisotropic elasticity, Philos. Mag. 7, 625-646 (1958)

[3] D. M. Barnett and L. A. Swanger, The elastic energy of a straight dislocation in an infinite anisotropic elastic medium, Phys. Status Solidi B 48, 414-428 (1971)

[4] D. M. Barnett and J. Lothe, Synthesis of the sextic and the integral formalism for dislocation, Green functions and surface waves in anisotropic elastic solids, Phys. Norv. 7, 13-19 (1973)

[5] R. J. Asaro, J. P. Hirth, D. M. Barnett, and J. Lothe, A further synthesis of sextic and integral theories for dislocations and line forces in anisotropic media, Phys. Status Solidi B 60, 261-271 (1973)

[6] T. Mura, A note on the strain field of a dislocation line in anisotropic media, Phys. Status Solidi B 70, K1-K6 (1975)

[7] D. M. Barnett and J. Lothe, Line force loadings on anisotropic half-spaces and wedges, Phys. Norv. 8, 13-22 (1975)

[8] P. Chadwick and G. D. Smith, Foundations of the theory of surface waves in anisotropic elastic materials, Adv. Appl. Mech. 17, 303-376 (1977)

[9] J. Braekhus and J. Lothe, Dislocations at and near planar interfaces, Phys. Status Solidi B 43, 651-657 (1971)

[10] D. M. Barnett and J. Lothe, An image force theorem for dislocations in anisotropic bicrystals, J. Phys. F 4, 1618-1635 (1974)

[11] T. C. T. Ting, Line forces and dislocations in anisotropic elastic composite wedges and spaces, Phys. Status Solidi B, 14b, 81-90 (1988)

[12] C. H. Wu and Chao-Hsun Chen, Wedge-apex crack in an angularly inhomogeneous wedge, J. Appl. Mech., in press (1989)

[13] T. C. T. Ting, The anisotropic elastic wedge under a concentrated couple, Quart. J. Mech. Appl. Math., 41, 563-578 (1988)

[14] T. C. T. Ting, Some identities and the structure of $\mathbf{N}_{i}$ in the Stroh formalism of anisotropic elasticity, Quart. Appl. Math. 46, 109-120 (1988)

[15] H. O. K. Kirchner, Line defects along the axis of rotationally inhomogeneous media, Phil. Mag. A, 55, 537-542 (1987) 